\title{
Field evaluation of five-level heat dissipation models under PV array structure installed in the tropics
}

\begin{abstract}
The intermittent drawbacks due to fluctuating temperature factor inside photovoltaic (PV) cells have clearly affected the overall energy performance especially in stochastic weather conditions. Temperature element in the tropical regions is a crucial factor to be determined based on Standard Testing Condition (STC) and Nominal Operating Cell Temperature (NOCT) correlations. Based on the crucial implication of heat dissipation, this study shares some insights of five level heat contour covering the surrounding temperature, PV surface temperature, PV bottom temperature and 2-level of two feet height located under PV array. The field data in real-time approach has been brought up in line to support the energy balance modelling for PV applications with localized heat contour analysis using statistical evaluations. The regression analysis of the 3471 data sampling for the period of 5 days (7AM till 7 PM) produces very good results with correlation coefficient, $\mathrm{R} 2=0.97$.
\end{abstract}

Keyword: Heat dissipation; Linear regression; PV array; Tropical climate 